\title{
Disks with Special Properties of Densest Packings
}

\author{
Peter Schmitt \\ Institut für Mathematik, Universität Wien, A-1090 Wien, Austria
}

\begin{abstract}
A general method is used to construct disks with special packing properties. In particular, one of the examples shows that there are disks (starshaped unions of two convex sets) such that no periodic packing has maximal density.
\end{abstract}

\section{Preliminaries}

A subset of the Euclidean plane is called a disk if it is homeomorphic to the closed unit disk. A family $\mathscr{P}=\left\{D_{1}, D_{2}, \ldots\right\}$ of disks is a packing if the disks do not overlap, i.e., if their interiors are pairwise disjoint. We are concerned with packings of a (single) disk $D$ or, more precisely, of disks congruent to $D$.

The density of a packing with respect to some bounded (measurable) subset $S$ of the plane is the ratio of the area of that part of $S$ that is covered by the disks and of the area of $S$ itself. The density $d$ of a packing (if it exists) is the limit of the densities with respect to $C(r, P)$, where $r$ tends to infinity and $C(r, P)$ is the circle with radius $r$ and centre $P$. The supremum (maximum) over the densities of packings of a disk $D$ is denoted by $d^{*}(D)$. If the density of a packing of $D$ equals $d^{*}(D)$, then it is called a densest packing of $D$.

A symmetry of a packing $\mathscr{P}$ is an isometry that maps each disk $D_{i}$ onto some disk $D_{j}$. A packing is called periodic if among its symmetries there are two nonparallel translations. It is a lattice packing if two translations generate a group of symmetries that acts transitively on the disks. In a packing of $D$, two disks have the same aspect if one is a translate of the other.

A tiling $\mathscr{T}=\left\{T_{1}, T_{2}, \ldots\right\}$ is a family of disks (now called tiles) which is a packing and covers the plane (i.e., every point of the plane belongs to some tile). Of course, the density of a tiling always equals 1.

A set $\left\{T_{1}, \ldots, T_{n}\right\}$ of tiles is called a set of prototiles. It admits a tiling if there is a tiling such that each of its tiles is congruent to one of the prototiles. A set of prototiles is called aperiodic if it admits a tiling but does not admit a periodic tiling. 


\section{Introduction}

In the theory of packings an interesting problem deals with the question whether among the densest packings of a disk $D$ there are packings with certain symmetry properties. For instance, if $D$ is convex and centrally symmetric, then maximal density can be obtained by a lattice packing. On the other hand, as tilings by congruent convex pentagons show, this is not true in general. A similar situation arises if only packings of translates of $D$ are considered: if $D$ is a convex disk, then maximal density can always be obtained by a lattice packing, but not necessarily so if $D$ is only assumed to be starshaped. (See [1] and [4].)

The general construction described below is adapted from [5] and allows us to construct some related examples. In particular, it yields a starshaped disk $D$ - which is the union of only two convex sets - such that all its densest packings are nonperiodic, or, equivalently, such that no periodic packing of $D$ has maximal density. In this connection it should be noted that, since the density of a packing is defined asymptotically, from any symmetric packing an asymmetric packing with the same density can easily be obtained: it suffices to change the packing locally, for instance simply by removing a single disk. Consequently, the converse statement is not true: There is no disk such that all its densest packings are periodic.

\section{Construction of the Disks}

The basis of the construction are sets $\left\{H_{1}, \ldots, H_{n}\right\}$ of prototiles of the following type: The prototiles are regular hexagons of equal size with modified edges (see Fig. 1). The shapes of the edges define the matching conditions which determine if, in a tiling, one edge can be fitted against another without gaps or overlaps. Without loss of generality, it can be assumed that these modifications are restricted to sufficiently small parts at the centres of the edges.

From this set of $n$ hexagons a regular $6 n$-gon $D$ (with modified edges) is obtained: $D$ is the intersection of concentric copies of the hexagons $H_{i}(i=1, \ldots, n)$

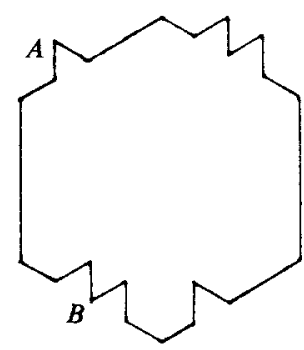

(a)

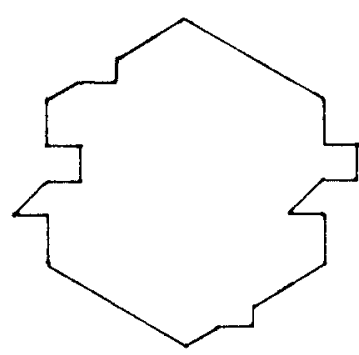

(b)

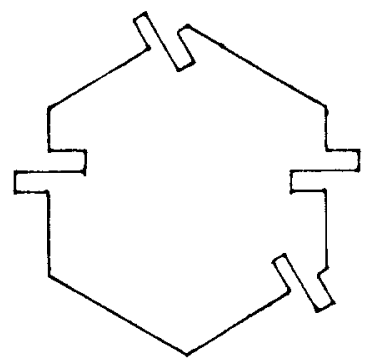

(c)

Fig. 1. A set of three hexagons with modified edges. Without alterations, neither (a) nor (b) can be used for the construction of $D$ since edges which do not match can, nevertheless, be fitted against each other without overlaps, e.g., $A$ against $B$. The hexagon (c) shows how an admissable modification may look. 


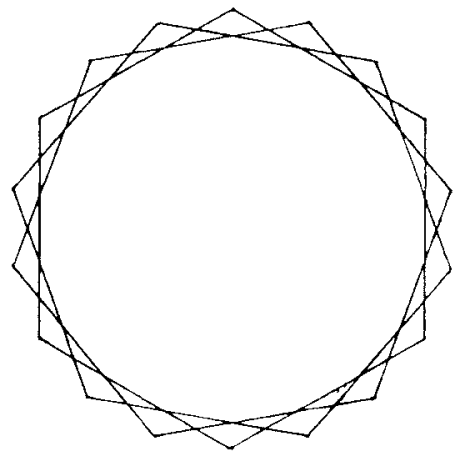

(a)

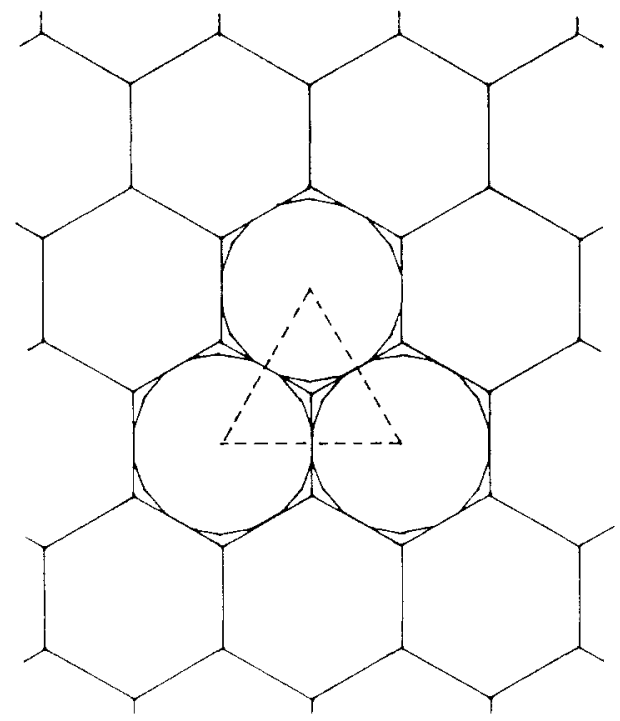

(b)

Fig. 2. (a) Construction of $D$ as an intersection of hexagons. (b) Correspondence between the tilings by hexagons and the packings of $D$.

where $H_{i}$ is rotated through $\pi / 3 n$ with respect to $H_{i-1}$, as shown in Fig. 2(a). Thus, for any $k, 1 \leq k \leq n$, there is an induced embedding of $D$ into $H_{k}$ such that six edges of $D$ are subsets of the edges of $H_{k}$. As already mentioned, we may assume that the matching conditions are realized on these subsets. By $r$ we denote the radius of the circle inscribed into the regular $6 n$-gon $D$. It is equal to that of the original hexagons. The radius of the circumscribed circle is denoted by $R$. (In both cases the effect of the modifications is neglected. In fact, it may be assumed that the indentations do not meet the inscribed circle, and that the projections do not exceed the circumscribed circle, but this assumption is not necessary.) Obviously, to each tiling admitted by the set of hexagons there corresponds a packing of $D$ in which the copies of $D$ touch along matching edges of the $6 n$-gon. To each tile of the tiling there corresponds some aspect of $D$, determined by the embedding of $D$ into the tile. It is uniquely determined if the tile has no symmetry. If the tile has some symmetries, then several distinct embeddings are possible if $D$ does not have the same symmetries as the tile (as, in general, is not the case). Conversely, if in a packing the disks touch along matching edges, then this packing corresponds to a tiling: It can be obtained by replacing each copy of $D$ by that prototile that corresponds to the aspect of the disk $D$. This tiling, of course, is uniquely determined. (See Fig. 2(b).)

Furthermore, we note that in these packings the distance of the centres of two touching disks always equals $2 r$, i.e., the inscribed circles touch. Moreover, if the realization of the matching conditions is chosen accordingly, then this property characterizes the packings which are derived from tilings. 

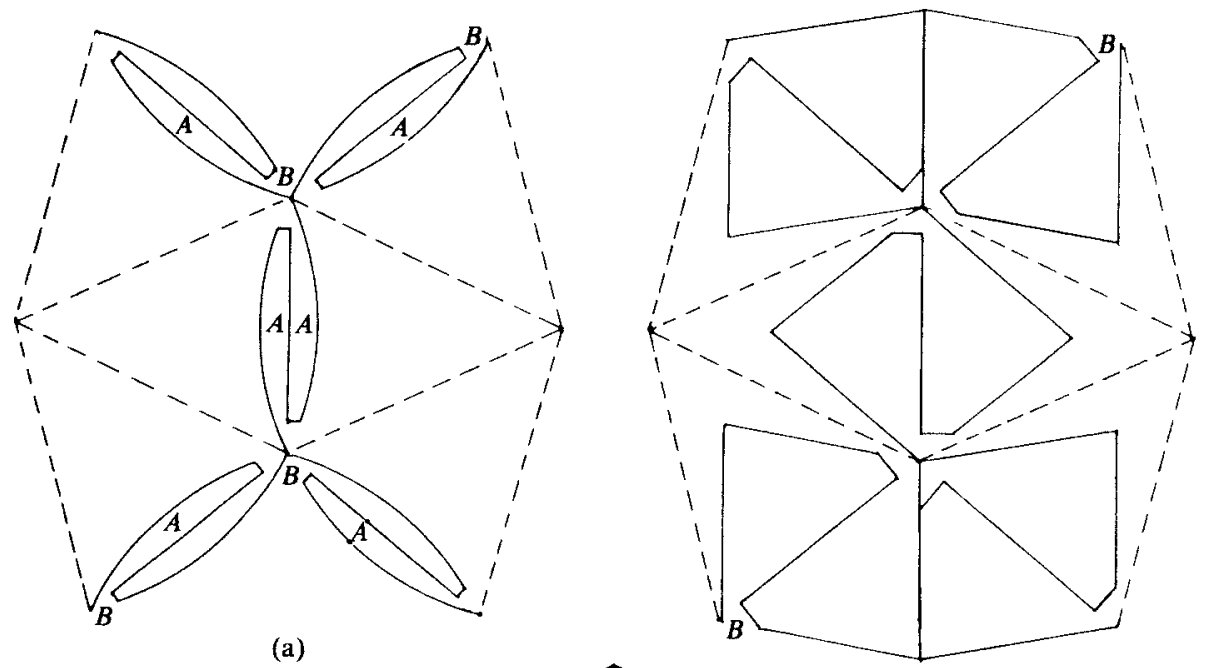

(a)

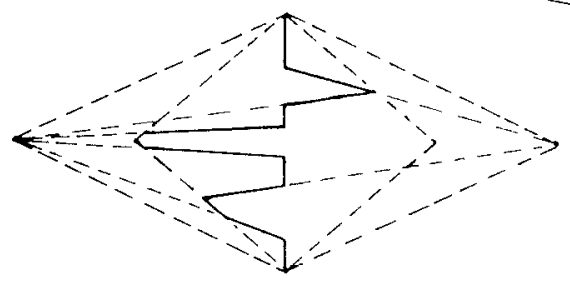

(b)

(c)

Fig. 3. Realization of the matching conditions. (a) and (b) Two general shapes of edges which assure maximal distance of the centres if the disks do not touch along matching edges. The modifications are realized at that part of the edge marked $A$. The gap (marked $B$ ) can be made arbitrarily small. Three sectors of each of the two disks are shown. (c) A realization of the matching conditions which yields starshaped disks.

In packings, gaps are allowed, but not overlaps. Therefore, a condition sufficient for our purpose is the assumption that if the distance of two disks is less or equal than $2 r$, then either the disks touch along matching edges or the disks overlap. To guarantee this, we assume that each edge which is not a straight line is provided with at least one projection, and that this projection is at a location where only the matching side has an indentation.

If the matching conditions are realized as indicated in Fig. 3(a) and (b) then, in any packing, the distance of two centres is either equal to $2 r$ (if matching edges interlock) or $\geq 2 R-\varepsilon$, where $\varepsilon$ is arbitrarily small since the gap (at $B$ in the figures) can be made sufficiently small. In these two cases edges cannot interlock if one disk is the mirror image of the other. Therefore, if this type of edges is used, then, if necessary, mirror images of hexagons have to be added to the initial set of prototiles.

If the matching conditions are realized by projections and indentations, as shown in Fig. 3(c), then the resulting disk $D$ is starshaped. Furthermore, to simplify the argument, we assume that the indentations are chosen so small that they do not 
meet a circle slightly larger than the inscribed circle. Then again, in any packing, the distance of two centres is $\geq 2 r$, and it is easy to prove that it is equal to $2 r$ if and only if the two disks touch along matching edges: To see this, we assume that the circles inscribed into two disks touch in a point, say $P$. If $P$ lies on the edges of both disks then - by the choice of the modifications - the disks do not overlap if and only if they match. If, for at least one of the disks, $P$ does not lie on an edge, then an edge of this disk cuts the inscribed circle of the other disk. Therefore, those parts of the disks that are, by assumption, not effected by indentations overlap, and consequently the two disks overlap, as well.

As a more careful construction shows, we can do even better: It is possible to use disks which are not only starshaped, but which are the union of only two convex sets. In exchange for this, the difference between packings which are derived from the tiling and other packings becomes even smaller.

As in the previous cases, the sectors of the disk are constructed independently of each other. Each sector will be the union of two convex sectors. To ensure that these convex sectors can be pasted together to form two convex disks we proceed as follows:

Let $C A B$ be a typical sector, where $C$ denotes the centre of the $6 n$-gon, and $A$ and $B$ are the endpoints of one side. Let $E$ and $F$ be points on $A C$ and $B C$, respectively, where $A E=B F=\delta$ for some small $\delta>0$. Let $D E$ be orthogonal to $A C$, and let $D F$ be orthogonal to $B C$. The convex sectors to be constructed will all contain the triangle $C E F$ and be subsets of the deltoid $C E D F$. Clearly, this condition ensures that any disk assembled from $6 n$ such sectors is a convex set. (See Fig. 4.)

The matching conditions are represented by a single triangular projection and by a matching indentation on the corresponding edge. In order to avoid a projection being fitted against an indentation with a wider opening, wider indentations are chosen to be less deep. Furthermore, if the projections (and indentations) are chosen to be asymmetric, a mirror image of the edge will not fit (see Fig. 5). Since two edges with indentations can always be placed against each other, a narrow strip (width $\delta^{\prime}$ for some small $\delta^{\prime}$ ) is added to each such edge. Of course, a corresponding strip has to be removed from all edges with projections.

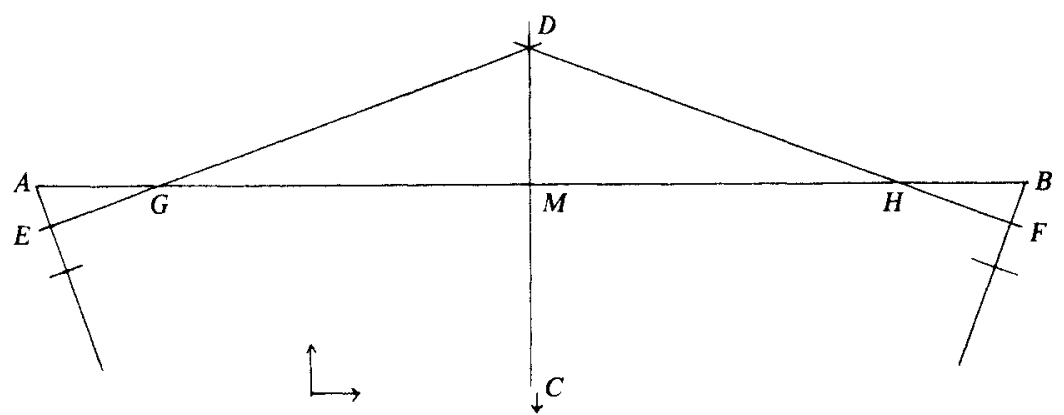

Fig. 4. A typical sector. The modifications are made such that the set lies between $C E F$ and $C E D F$. 


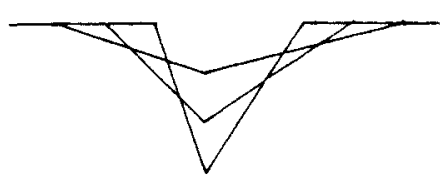

(a)

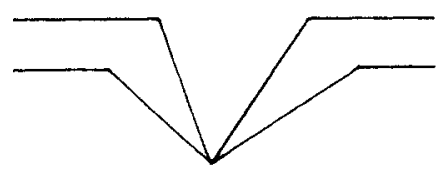

(c)

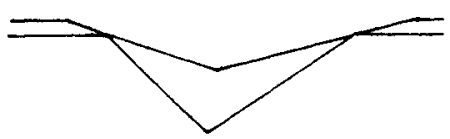

(e)

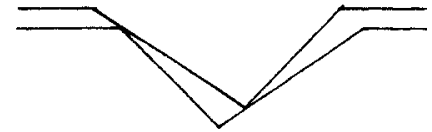

(b)

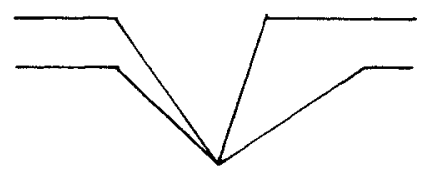

(d)

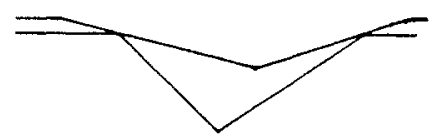

(f)

Fig. 5. Representation of the matching conditions. (a) Three different indentations. (b) The mirror image of the matching projection does not fit. (c)-(I) The "wrong" projections do not fit.

Projections and indentations, as described, can be realized as the union of two convex sets: Choose two points, $P$ and $Q$, on $A B$. The lines $Q E$ and $P F$ meet in $N$. Let $M N$ be orthogonal to $P Q$ where $M$ lies on $P Q$. Choose $X$ on $G P, Z$ on $Q H$ ( $G$ and $H$ are the intersections of $D E$ and $D F$, respectively, with $A B$ ), and $Y$ on $M N$. Then $C E G X Y F, C E Y Z H F, C^{\prime} F^{\prime} H G E^{\prime}$, and $C^{\prime} F^{\prime} Z Y X E^{\prime}$ are convex polygons $\left(C^{\prime}, E^{\prime}, F^{\prime}\right.$ lie symmetric to $C, E$, and $F$, with respect to $A B$ ). Finally, adding a strip of width $\delta^{\prime}$ (i.e., drawing a line parallel to $A B$ ) yields the final convex sets: $C E G^{\prime} X^{\prime} Y F, C E Y Z^{\prime} H^{\prime} F, C^{\prime} F^{\prime} H^{\prime \prime} G^{\prime \prime} E^{\prime}$, and $C^{\prime} F^{\prime} Z^{\prime} Y X^{\prime} E^{\prime}$. (See Fig. 6.)

If $\delta$ is small enough, if the indentations are chosen sufficiently small, and if $\delta^{\prime}$ is sufficiently small with respect to the projections, then the disks have the desired property: The distance of the centres $C_{1}$ and $C_{2}$ of two nonoverlapping disks is always $\geq 2 r$. It equals $2 r$ if and only if the disks touch along matching edges, or else it is $\geq 2 r+\varepsilon$, for some small $\varepsilon>0$. To see this the same argument as in the previous case can be used if, again, we assume that indentations are not in the middle of the edges and do not meet a circle slightly larger than the inscribed circle. Again, this assumption is not necessary though it simplifies the argument.

These observations lead to the following proposition:

A packing of the disk $D$ is derived from a tiling by $H_{1}, \ldots, H_{n}$ if and only if each inscribed circle touches six other inscribed circles. Each such packing of $D$ has maximal density.

The first assertion follows immediately from the properties of $D$. The second assertion is an easy consequence: Denote by $\lambda$ the ratio of the areas of the disk $D$ 


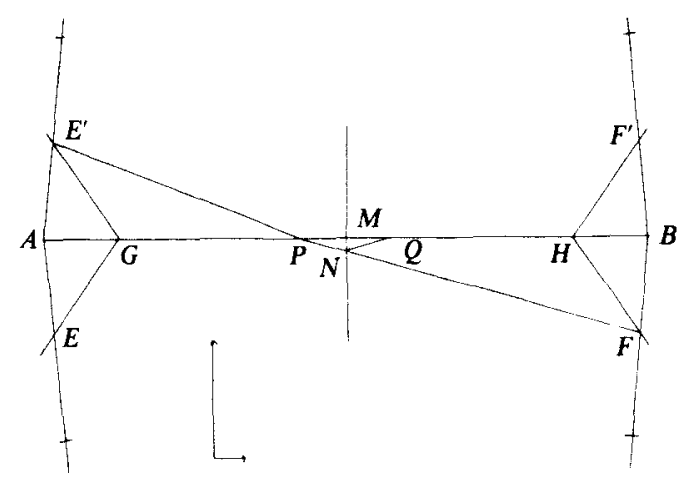

(a)

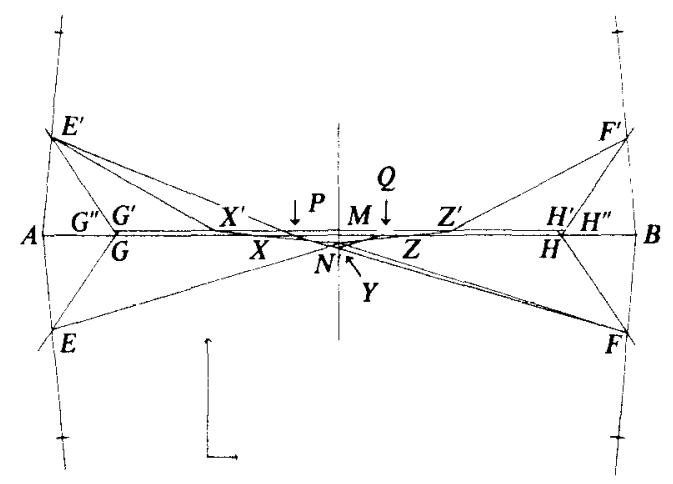

(b)

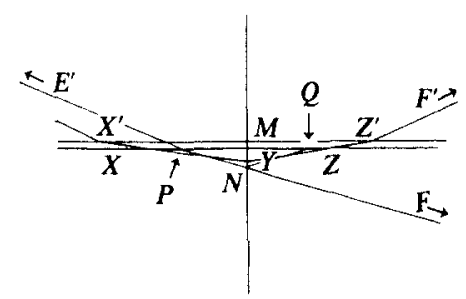

(c)

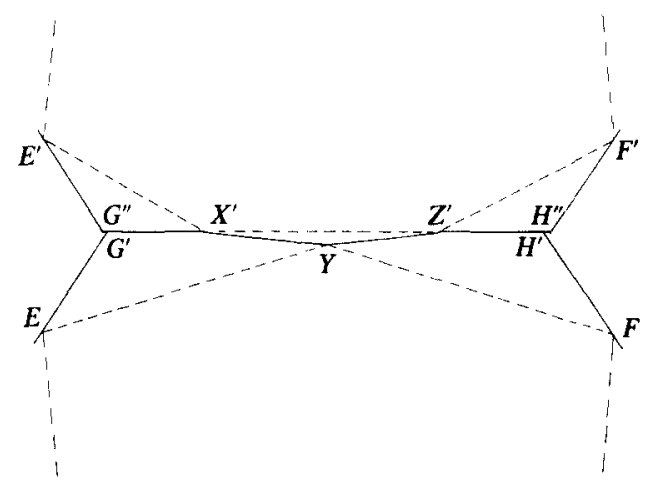

(d)

Fig. 6. Construction of the edge. (a) The points of reference. The vertical scale has been changed (factor 4 compared with Fig. 4). Note that $M$ need not be the central point of $A B$. (b) Construction of two matching edges. (c) A magnification of the central part of (b). (d) Two matching edges, and the corresponding four convex sets: $C E G^{\prime} X^{\prime} Y F, C E Y Z^{\prime} H^{\prime} F, C^{\prime} F^{\prime} H^{\prime \prime} G^{\prime \prime} E^{\prime}$, and $C^{\prime} F^{\prime} Z^{\prime} Y X^{\prime} E^{\prime}$. 
and of the inscribed circle. Then the density of any packing of $D$ equals $\lambda$-times the density of the packing formed by the inscribed circles. Since it is well known that the circle packings that occur have maximal density (they are, in fact, the densest lattice packing) the assertion follows.

\section{Applications}

\subsection{A Disk such that No Periodic Packing Has Maximal Density}

The construction starts with any aperiodic set of hexagons. Such a set can be adapted from one of the well-known aperiodic sets of Wang tiles. The smallest such set that is known contains 16 tiles (see p. 595 of [3]). Wang tiles are square prototiles with modified edges such that in a tiling each prototile appears in only one aspect, i.e., such that only translates of the prototiles can be used. Therefore, each set of Wang tiles $W_{1}, \ldots, W_{n}$ can be transformed into an equivalent set of (asymmetric) hexagonal prototiles $H_{1}, \ldots, H_{n}$ : Four edges of the hexagon $H_{i}$ have the same shape as the edges of the corresponding square $W_{i}$. The fifth and the sixth sides (two opposite sides) are the same on each hexagon. They match each other, and are different from all the other edges (see Fig. 7).

We claim that any disk $D$ obtained by applying the construction described in the previous section has the desired property.

To prove this we consider an arbitrary packing of $D$ and replace each copy by its inscribed circle. It is known from the theory of circle packings that there is a triangulation such that each centre of a circle is a vertex of the triangulation, and such that in no triangle does a circle cut the side opposite its centre. The density of the packing with respect to such a triangle is maximal and equal to the density of the densest circle packing if and only if it is determined by three touching circles (see Fig. 8(b)) (see [2]).

We now assume that the packing of $D$ is periodic. Then, clearly, the same is true for the induced circle packing. On the other hand, since the initial set of 16 hexagons is aperiodic, the packing of $D$ (a 96-gon) cannot correspond to a tiling
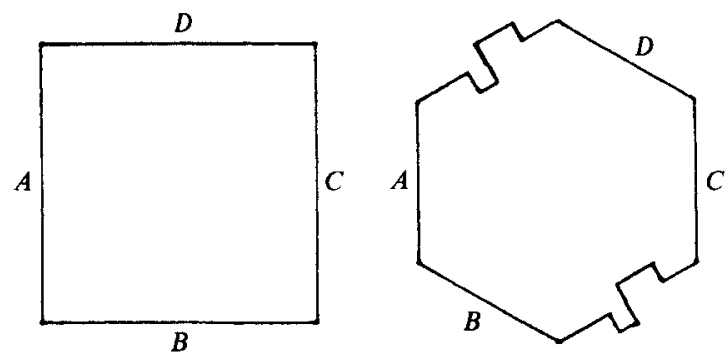

Fig. 7. Construction of a set of hexagons equivalent to a set of Wang tiles. Edges with the same label have the same shape. 


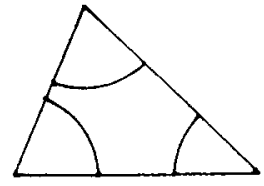

(a)

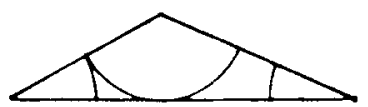

(c)

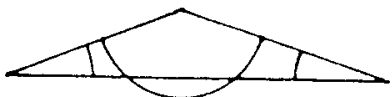

(d)

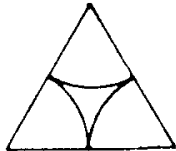

(b)

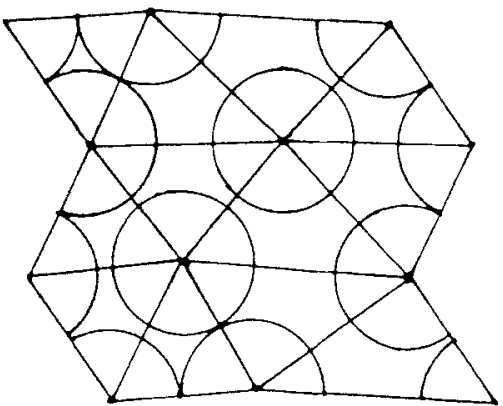

(e)

Fig. 8. The triangulation corresponding to a circle packing. (a)-(c) Admissable triangles. (d) Forbidden triangle. It can be avoided. (e) A fundamental region and its triangulation.

because then the tiling necessarily would have to be periodic, too. Consequently, the triangulation cannot be the triangulation by regular triangles because this characterizes the densest lattice packing of circles. We now choose a family of triangles whose union is a fundamental region for the group of translations. Among them there must be at least one triangle with respect to which the density is not maximal. Therefore, the density with respect to the fundamental region is not maximal. But, because of periodicity, this density equals the density of the packing.

\subsection{A Disk Which Has Precisely $n$ Densest Periodic Packings}

If the initial set of hexagonal prototiles admits precisely $n$ (periodic) tilings, then an argument similar to that used in the previous example shows that any further periodic tiling cannot have maximal density. Sets of prototiles with the desired property are easily constructed: We may, for instance, take $n$ asymmetric hexagons such that each hexagon admits precisely one tiling (consisting of translates of the hexagon), but no other tilings (using two or more of the hexagons) are possible since edges of distinct prototiles do not match. (See Fig. 1 for an example with $n=3$.) In the case just described each of the densest periodic packings is a lattice packing since, in each of these packings, $D$ occurs in just one aspect. But, using other sets of hexagons, it is easy to find disks such that any prescribed number of aspects occurs in the densest periodic packings. Moreover, the number of aspects may be different in each of the packings. 


\subsection{A Disk Which Locally Can Be Packed Denser than Globally}

It is easy to construct sets of prototiles which admit tilings of some (finite) subset of the plane (a patch), but do not admit a tiling: Consider some patch of regular hexagons. If each edge (of the tiling) is replaced by an edge which is different to all other edges, a large number of prototiles are generated. Each of these prototiles occurs only once, and any patch admitted by them is congruent to a subset of this patch. The disk $D$ corresponding to this set of prototiles has the following packing property: there are packings of $D$ into subsets of the plane (namely, those corresponding to a patch) such that the inscribed circles of touching disks touch. Therefore, the triangles in the corresponding triangulation are all optimal (smallest regular triangles, as shown in Fig. 7(b)). But, since the prototiles do not admit a tiling, there is no packing of $D$ (into the whole plane) which has this property. In this sense, it can be said that $D$ can be packed more densely into a subset of the plane than into the plane.

\section{Acknowledgment}

I want to thank L. Danzer for his interest in this work, and for suggesting that the union of two convex sets might suffice.

\section{References}

1. A. Bezdek and G. Kertész, Counterexamples to a packing problem of L. Fejes Tóth. In: Intuitive Geometry (Siófok 1985) (Coll. Math. Soc. J. Bolyai, Vol. 48), pp.29-36. North-Holland, Amsterdam, 1987.

2. L. Fejes Tóth, Reguläre Figuren. Akadémiai Kiadó, Budapest, 1965.

3. B. Grünbaum and G. C. Shephard, Tilings and Patterns. Freeman, New York, 1986.

4. A. Heppes, On the density of translates of a domain. MTA SZTAKI Közlemények 36 (1987), 93-97.

5. P. Schmitt, An aperiodic triple of prototiles. In: Topics in Combinatorics and Graph Theory (ed. by R. Bodendiek and R. Henn), pp. 627-633. Physica-Verlag, Heidelberg, 1990.

Received September 1, 1988. 